\title{
Genome-wide identification and characterization of long non-coding RNAs conferring resistance to Colletotrichum gloeosporioides in walnut (Juglans regia)
}

Shan Feng ${ }^{1+}$, Hongcheng Fang ${ }^{1,2,3+}$, Xia Liu ${ }^{1,4}$, Yuhui Dong ${ }^{1}$, Qingpeng Wang ${ }^{1}$ and Ke Qiang Yang ${ }^{1,2,3^{*}}$ (D)

\begin{abstract}
Background: Walnut anthracnose caused by Colletotrichum gloeosporioides (Penz.) Penz. and Sacc. is an important walnut production problem in China. Although the long non-coding RNAs (IncRNAs) are important for plant disease resistance, the molecular mechanisms underlying resistance to C. gloeosporioides in walnut remain poorly understood.

Results: The anthracnose-resistant F26 fruits from the B26 clone and the anthracnose-susceptible F423 fruits from the 4-23 clone of walnut were used as the test materials. Specifically, we performed a comparative transcriptome analysis of F26 and F423 fruit bracts to identify differentially expressed LncRNAs (DELs) at five time-points (tissues at $0 \mathrm{hpi}$, pathological tissues at $24 \mathrm{hpi}, 48 \mathrm{hpi}, 72 \mathrm{hpi}$, and distal uninoculated tissues at $120 \mathrm{hpi}$ ). Compared with F423, a total of 14,525 DELs were identified, including 10,645 upregulated IncRNAs and 3846 downregulated IncRNAs in F26. The number of upregulated IncRNAs in F26 compared to in F423 was significantly higher at the early stages of C. gloeosporioides infection. A total of 5 modules related to disease resistance were screened by WGCNA and the target genes of IncRNAs were obtained. Bioinformatic analysis showed that the target genes of upregulated IncRNAs were enriched in immune-related processes during the infection of C. gloeosporioides, such as activation of innate immune response, defense response to bacterium, incompatible interaction and immune system process, and enriched in plant hormone signal transduction, phenylpropanoid biosynthesis and other pathways. And 124 known target genes for 96 hub IncRNAs were predicted, including 10 known resistance genes. The expression of 5 IncRNAs and 5 target genes was confirmed by qPCR, which was consistent with the RNA-seq data.
\end{abstract}

Conclusions: The results of this study provide the basis for future functional characterizations of IncRNAs regarding the C. gloeosporioides resistance of walnut fruit bracts.

Keywords: Walnut (Juglans regia L.), Colletotrichum gloeosporioides (Penz.) Penz. And Sacc., IncRNA, WGCNA

\footnotetext{
* Correspondence: yangwere@126.com

${ }^{\dagger}$ Shan Feng and Hongcheng Fang contributed equally to this work.

'College of Forestry, Shandong Agricultural University, Tai'an 271018,

Shandong Province, China

${ }^{2}$ State Forestry and Grassland Administration Key Laboratory of Silviculture in

the Downstream Areas of the Yellow River, Tai'an 271018, Shandong

Province, China

Full list of author information is available at the end of the article
}

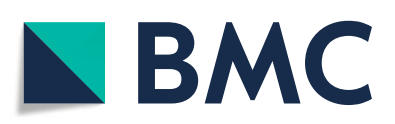

(- The Author(s). 2021 Open Access This article is licensed under a Creative Commons Attribution 4.0 International License, which permits use, sharing, adaptation, distribution and reproduction in any medium or format, as long as you give appropriate credit to the original author(s) and the source, provide a link to the Creative Commons licence, and indicate if changes were made. The images or other third party material in this article are included in the article's Creative Commons licence, unless indicated otherwise in a credit line to the material. If material is not included in the article's Creative Commons licence and your intended use is not permitted by statutory regulation or exceeds the permitted use, you will need to obtain permission directly from the copyright holder. To view a copy of this licence, visit http://creativecommons.org/licenses/by/4.0/. The Creative Commons Public Domain Dedication waiver (http://creativecommons.org/publicdomain/zero/1.0/) applies to the data made available in this article, unless otherwise stated in a credit line to the data. 


\section{Background}

Walnut (Juglans regia L.) is a diploid tree species $(2 \mathrm{n}=$ 32 ), with approximately $667 \mathrm{Mb}$ per $1 \mathrm{C}$ genome and an N50 size of 464,955 (based on a genome size of 606 Mbp) [1]. It is an ecologically important 'woody oil' tree species worldwide [2], and its kernel is a rich source of nutrients with health benefits for humans [3]. The peptides extracted from walnut seeds have antioxidant and anticancer activities and have the protective effects on the oxidative damage induced by $\mathrm{H}_{2} \mathrm{O}_{2}$ [4]. Recent advances in biotechnology and genomics show potential to accelerate walnut breeding, such as gamma-irradiated pollen inducing haploid walnut plants [5], constructing the novel Axiom J. regia $700 \mathrm{~K}$ SNP array [6], and combining different assemblies to obtain the optimal version [7]. Walnut anthracnose caused by Colletotrichum gloeosporioides (Penz.) Penz. and Sacc can cause leaf scorch or defoliation and fruit gangrene, which is currently the disastrous disease in walnut production [8]. Due to the long incubation period of anthracnose, the concentrated onset time, and the strong outbreak, the use of chemical fungicides is still the main method of disease control [9]. The C. gloeosporioides lifestyle transitions associated with the infection of the host include the following three stages: attachment, biotrophy, and necrotrophy [10]. The pathogen of C. gloeosporioides in walnut overwinters in the diseased part with mycelium, and begins to move when the temperature reaches 11$15^{\circ} \mathrm{C}$ in the following spring [11]. Specifically, the formation of adherent cells is critical for fungal development during the $C$. gloeosporioides infection [12]. In a previous study, LAC2 was revealed to contribute to the formation of adherent cells to enhance the pathogenicity of C. gloeosporioides [13]. However, it is unclear how walnuts recognize and resist infections by $C$. gloeosporioides, and the regulatory network of hub and peripheral genes underlying the resistance of walnuts to C. gloeosporioides remains uncharacterized. Therefore, elucidating the molecular basis of this resistance mechanism is imperative for the breeding of walnut resistant to $C$. gloeosporioides [8, 14, 15].

Long non-coding RNA (lncRNA) is a type of RNA comprising 200-1,000,000 nt and structural characteristics similar to those of mRNA, but it does not encode a protein [16]. The lncRNAs were initially considered to be the transcription 'noise' of protein-coding genes, and were often ignored in transcriptome analyses [17]. However, the continuous development of sequencing technologies and transcriptome analyses has revealed that many lncRNAs in Arabidopsis thaliana [18], Triticum aestivum [19], Zea mays [20], and other plant species are related to stress responses, morphological development, and fruit maturation. For example, a heat-responsive lncRNA (TCONS_00048391) is an eTM for bra-miR164a and may be a competing endogenous RNA (ceRNA) for the target gene NAC1 (Bra030820), with effects on bra-miR164a expression in Chinese cabbage (Brassica rapa ssp. chinensis) [21]. Qin et al. confirmed that the DROUGHT INDUCED lncRNA regulates plant responses to abiotic stress by modulating the expression of a series of stress-responsive genes [22]. In A. thaliana, two lncRNAs, COOLAIR and COLDAIR, are associated with FLOWERING LOCUS C and play an crucial role in vernalization [23, 24].

Many recent studies have proved that lncRNAs are important for plant-pathogen interactions. A role for nine hub lncRNAs and 12 target genes in the resistance of Paulownia tomentosa to witches'broom was uncovered via a high-throughput sequencing experiment, and their functions were analyzed with an RNA-IncRNA co-expression network model [25]. In tomato (Solanum lycopersicum), the lncRNA16397-GRX21 regulatory network reportedly decreases the reactive oxygen species content and cell membrane damage to enhance the resistance to $P$. infestans [26]. Moreover, the involvement of the WRKY1lncRNA 33,732- $\mathrm{RBOH}$ module in regulating $\mathrm{H}_{2} \mathrm{O}_{2}$ accumulation and resistance to $P$. infestans was determined based on a comparative transcriptome analysis [27]. In cotton (Gossypium spp.), a functional analysis demonstrated that a lack of two hub lncRNAs, GhlncNATANX2 and GhlncNAT-RLP7, enhances seedling resistance to Verticillium dahliae and Botrytis cinerea, possibly because of the associated upregulated expression of LOX1 and LOX2 [28]. In wheat (Triticum aestivum L.), IncRNAs have a tissue-dependent expression pattern that can respond to powdery mildew infections and heat stress [29]. Additionally, four kinds of lncRNAs have important effects on Puccinia striiformis infections [30]. However, there are no reports regarding the role of lncRNAs in the walnut fruit resistance to anthracnose.

In this study, Illumina HiSeq 4000 sequencing was used to analyze the disease-resistant (F26) and susceptible (F423) fruit bracts at different C. gloeosporioides infection stages. The number and characteristics of lncRNAs were analyzed. Additionally, the hub lncRNAs related to disease resistance were screened and functionally analyzed to predict the role of lncRNAs in walnut fruit bract resistance to anthracnose. To the best of our knowledge, this is the first report on walnut lncRNAs and their biological functions related to fruit bract resistance to C. gloeosporioides. Our data may be a useful resource for clarifying the regulatory functions of lncRNAs influencing walnut fruit resistance to C. gloeosporioides.

\section{Results}

Symptoms and physiological changes of walnut fruit infected by C. gloeosporioide

The resistant (F26) and susceptible (F423) fruit bracts were infected by C.gloeosporioide, the fruit bracts of 
F423 showed obvious symptoms at $48 \mathrm{hpi}$; the diseaseresistant fruit F26 at 72 hpi. The susceptible samples showed obvious C.gloeosporioide conidial at $120 \mathrm{hpi}$ (Fig. 1a). During the infection, the activities of some enzymes and the content of hormones also changed correspondingly. Compared to the F423, the activities of chitinase, ROS-scavenging enzymes (catalase, CAT and superoxide dismutase, SOD) and the content of $\mathrm{H}_{2} \mathrm{O}_{2}$ in F26 were higher (Fig. 1b-e). The content of salicylic acid (SA) and jasmonic acid (JA) in F26 was significantly higher than that in F423, and reached a peak at 72hpi after infection (Fig. 1f, g).

\section{Whole genome identification of IncRNAs expressed in walnut fruit bracts}

To identify lncRNAs expressed in walnut fruits in response to C. gloeosporioides, we constructed $20 \mathrm{cDNA}$ libraries from the anthracnose-resistant and the anthracnosesusceptible walnut fruits at the following five infection stages: tissue at 0 hpi (hours post inoculation), infected tissue at 24, 48, and $72 \mathrm{hpi}$, and distal uninoculated tissue at 120 hpi (Additional file 1: Table S1). The libraries were sequenced with an Illumina HiSeq 4000 platform. A total of 265.4 $\mathrm{Gb}$ clean data were obtained, with an average of 13.27 Gb per library. Approximately $69.7 \%$ of the clean reads in all libraries were mapped to the walnut reference genome (Additional file 2: Table S2). The aligned transcripts were assembled, combined, and screened with the FEELnc software to obtain 22,336 lncRNAs (length $\geq 200$ nt, ORF coverage $<50 \%$, and potential coding score $<0.5$ ), including 18,403 unknown lncRNAs (23.97\%) and 3933 known lncRNAs (5.12\%) (Fig. 2a,b). The principal component analyses (PCA) revealed that the results at same infection point were parallel (Fig. 2c).

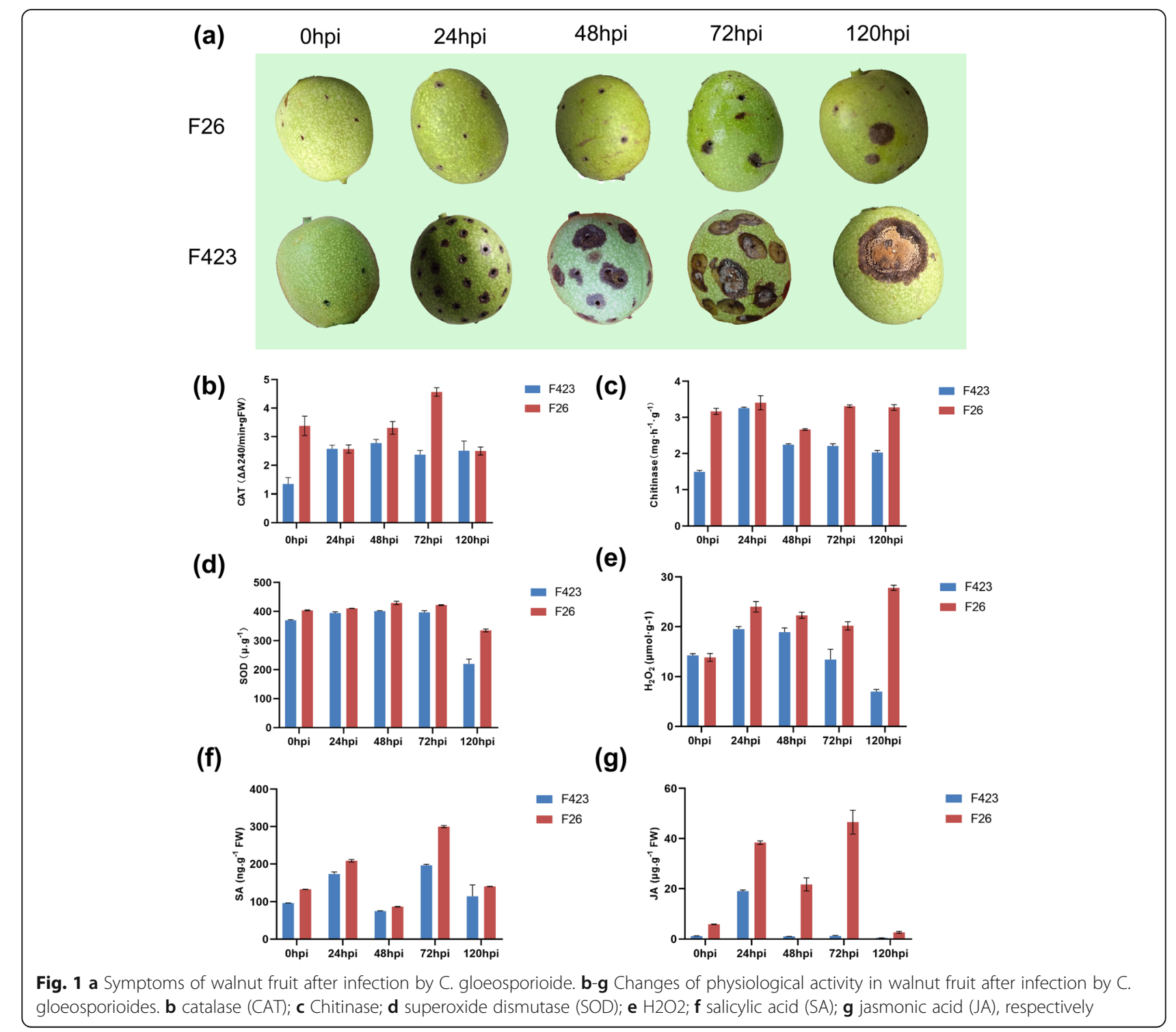



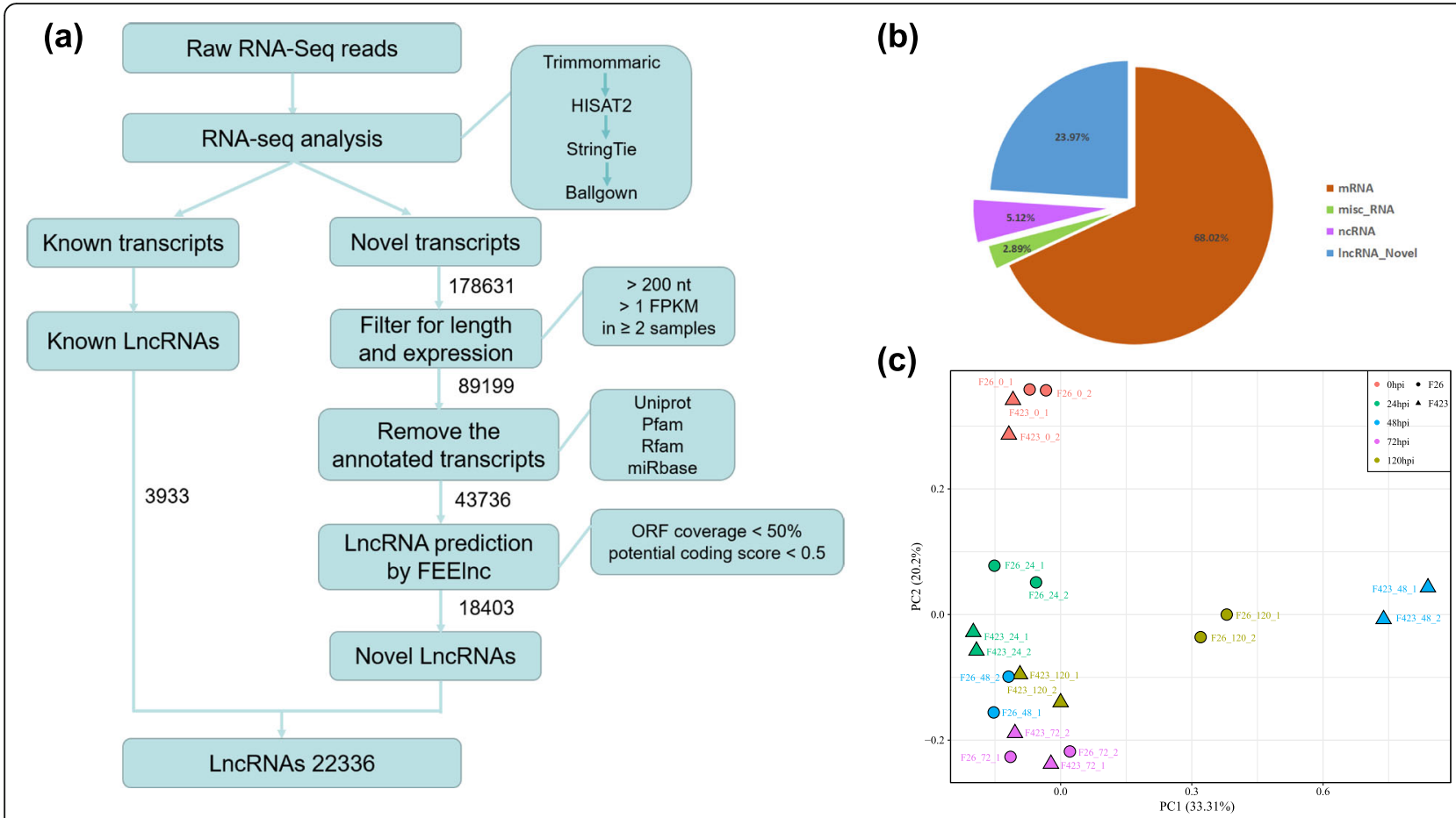

Fig. 2 Identification and characterization of long non-coding RNAs (IncRNAs) in walnut. a Bioinformatic pipeline for the identification of IncRNAs in walnut. Each step is described in detail in the Materials and Methods section. b Proportion of transcripts corresponding to IncRNAs. c Patterns of gene expression represented by principal component analysis (PCA) plots of normalized count matrices for walnut fruit bracts

\section{Characterization of walnut fruit bract IncRNAs}

A total of 58,369 mRNAs and 22,336 lncRNAs were obtained for the walnut fruit bracts (all samples combined) (Additional file 3: Table S3, Additional file 4:Table S4). The lncRNAs were characterized according to their locations relative to the partner RNA. A total of 40,429 (67.57\%) lncRNAs were located in intergenic regions (i.e., only $32.43 \%$ genic lncRNAs). Additionally, 19,767 (48.89\%) and $7302(37.63 \%)$ of the intergenic lncRNAs and genic lncRNAs were located in the antisense strand, respectively (Fig. 3a) (Additional file 5: Table S5). Most lncRNAs contained two or three exons, which differentiated them from mRNAs (Fig. 3c). Moreover, there was considerable diversity in the distribution of mRNA and lncRNA lengths (Fig. 3b). Furthermore, the expression level of most lncRNAs was significantly lower than that of mRNAs (Fig. 3d).

\section{Differentially expressed IncRNAs at various infection stages}

The lncRNAs that were differentially expressed between the disease-susceptible F423 fruits and the diseaseresistant F26 fruits at different C. gloeosporioides infection stages were analyzed. Compared with F423, a total of 14,525 DELs were identified, including 10,645 upregulated lncRNAs and 3846 down-regulated lncRNAs in F26. The number of upregulated and downregulated lncRNAs in the various comparisons were respectively as follows: 7668 and 1386 in the F26_0hpi vs F423_0hpi comparison; 6910 and 1165 in the F26_24hpi vs F423_ 24hpi comparison; 1721 and 1593 in the F26_48hpi vs F423_48hpi comparison; 898 and 1133 in the F26_72 hpi vs F423_72 hpi comparison; and 4711 and 550 in the F26_120 hpi vs F423_120 hpi comparison (Fig. 4a, b) (Additional file 6: Table S6). Additionally, compared with F423, a total of 34,007 differentially expressed mRNAs were identified, including 15,247 upregulated mRNAs and 13,198 downregulated mRNAs in F26. the number of upregulated and downregulated mRNAs in the various comparisons were respectively as follows: 6836 and 4622 in the F26_0 hpi vs F423_0 hpi comparison; 6392 and 3955 in the F26_24 hpi vs F423_24 hpi comparison; 3454 and 4347 in the F26_ 48 hpi vs F423_48 hpi comparison; 2709 and 3113 in the F26_72 hpi vs F423_72 hpi comparison; and 4976 and 3563 in the F26_120 hpi vs F423_120 hpi comparison (Fig. 4c, d) (Additional file 7: Table S7). These results revealed the similarities in the expression of lncRNAs and mRNAs. And the number of upregulated lncRNAs and mRNAs in F26 compared to in F423 was significantly higher at the early stages of C. gloeosporioides infection. 


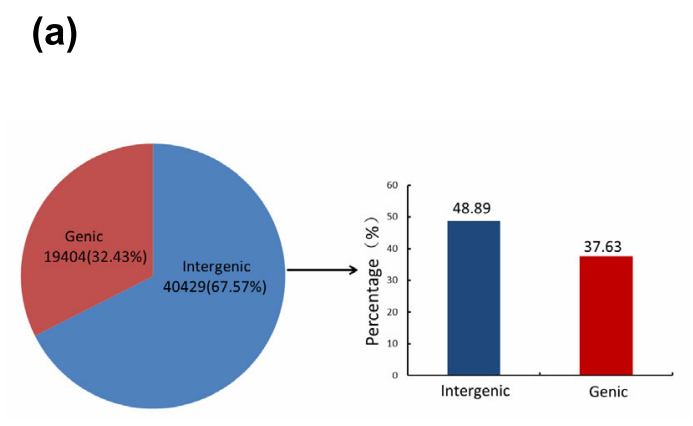

(b)

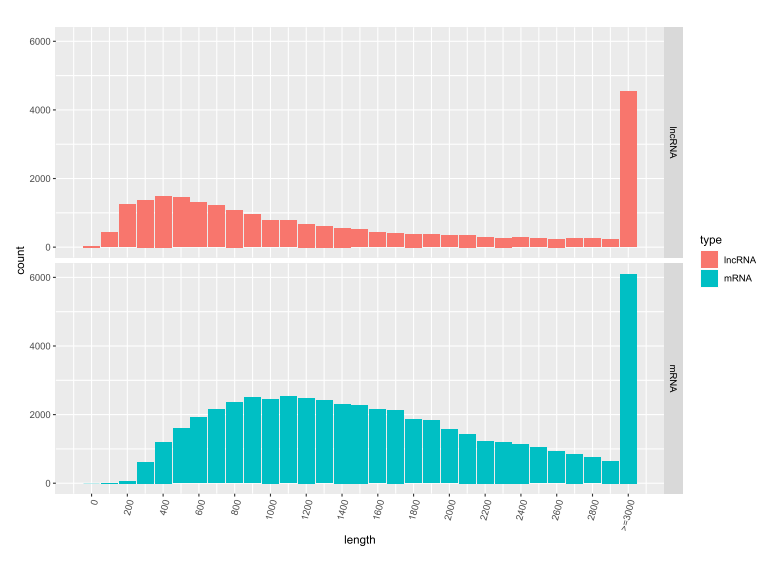

(c)

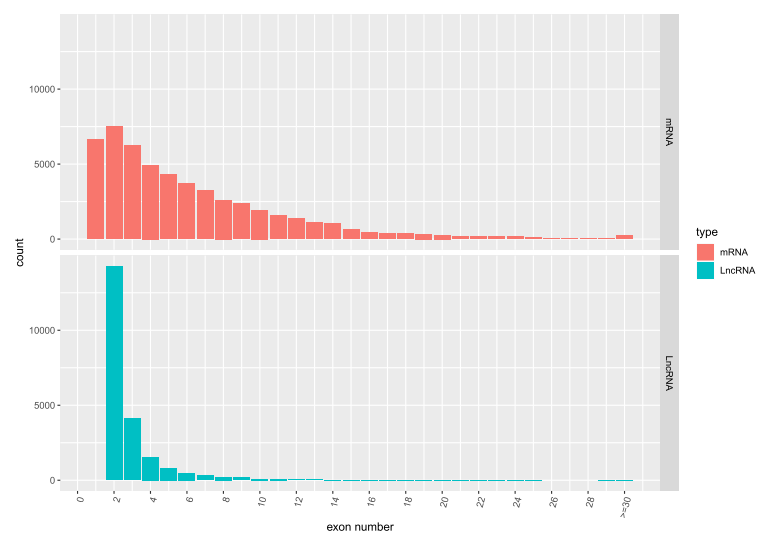

(d)

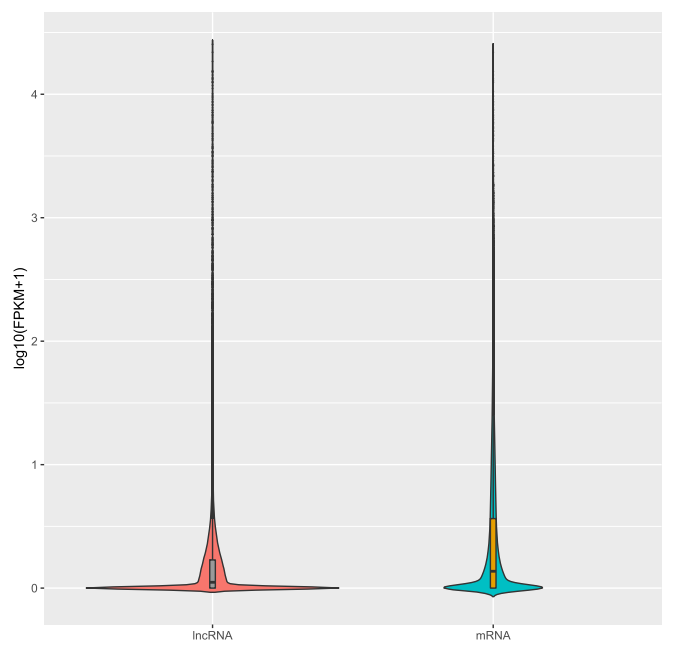

Fig. 3 Characteristics of walnut IncRNAs. a Proportion of IncRNAs that are located in intergenic and genic regions. $\mathbf{b}$ Length distribution of 22,336 newly predicted IncRNAs (red) and 58,369 protein-coding transcripts (blue). c Distribution of exon numbers in protein-coding genes (red) and IncRNA genes (blue). $\mathbf{d}$ Expression levels of protein-coding genes and IncRNA genes presented as $\log _{10}($ FPKM +1$)$ values

\section{Identification of co-expressed IncRNA modules}

To identify the hub lncRNAs and predict their potential target genes in trans-regulatory relationships, a weighted gene co-expression network analysis (WGCNA) was used to generate a correlation matrix of the expression levels of 10,645 upregulated lncRNAs and 15,247 upregulated mRNAs. A total of 19 expression modules were screened (Fig. 5a) (Additional file 8: Table_S8). The relationships between modules and the resistance traits of the walnut fruit bracts were analyzed and four significantly correlated modules $(|\mathrm{r}| \geq 0.8)$ were identified. The MEviolet module was correlated with F26_0hpi $(\mathrm{r}=0.95$, $p=9 \mathrm{e}-11$ ), which contains 406 lncRNAs and 1350 mRNAs. The MElightyellow module was correlated with F26_24hpi $(\mathrm{r}=0.86, p=1 \mathrm{e}-06)$, which contains 165 lncRNAs and 892 mRNAs. The MEbrown2 module was correlated with F26_48hpi $(\mathrm{r}=0.82, p=8 \mathrm{e}-0.86)$, which contains 128 lncRNAs and 224 mRNAs. The MEwhite module was correlated with F26_72hpi $(\mathrm{r}=0.81, \mathrm{p}=1 \mathrm{e}-$
05), which contains 111 lncRNAs and 378 mRNAs (Fig. 5c). Regarding F26_120 hpi, the rand $p$ value for the MEorange module was 0.73 and $3 \mathrm{e}-0.4$, respectively. The highest $r$ value (0.77) for F423 was calculated for the MEdarkseagreen module and F423_48hpi (Fig. 5b). And the MEorange module contains 76 lncRNAs and 227 mRNAs (Fig. 5c). These results suggested that lncRNAs are closely related to the disease resistance of walnut fruit bracts.

\section{Enrichment analysis of genes co-expressed with IncRNAs}

The GO and KEGG pathway databases were used to analyze the genes co-expressed with lncRNAs in each significant module and MEorange module. In the MEviolet module, a total of $208 \mathrm{GO}$ terms were assigned, including 106, 8 and $94 \mathrm{GO}$ terms in "biological process", "cellular component" and "molecular functions", respectively (Additional file 9:Table_S9). Among these enriched GO terms, most of them were 


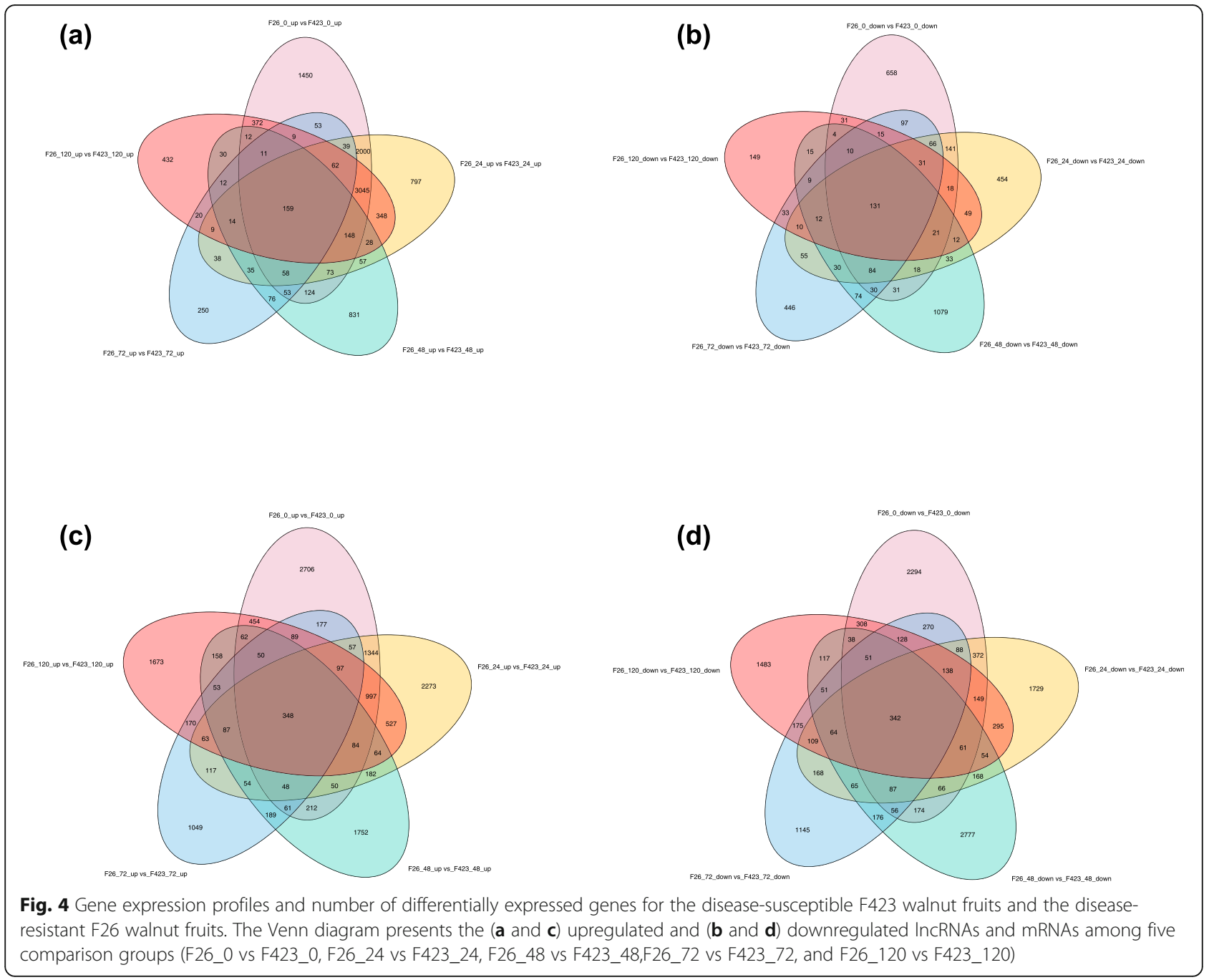

related to biosynthesis and gene expression regulation, and the ones related to plant immunity were "response to stimulus"(GO:0050896) (187 genes) and "cellular response to stimulus"(GO:0051716) (114 genes) (Fig. 6a). In total, 104 enriched KEGG pathways were identified, of which 30 pathways were significantly enriched in this module (Additional file 10: Table_S10). The top 30 significantly enriched pathways for target genes are mentioned in Fig. 7a. "Plant hormone signal transduction" (ko04075) (22 genes), "Fatty acid metabolism" (ko01212) (15 genes), "Fatty acid elongation" (ko00062) (12 genes), "Ribosome" (ko03010) (12 genes), and "Spliceosome" (ko03040) (11 genes) were the most significant KEGG pathways.

In the MElightyellow module, a total of $164 \mathrm{GO}$ terms were assigned, including 79, 16 and 69 GO terms in "biological process", "cellular component" and "molecular functions", respectively (Additional file 9: Table_S9). Among them, GO terms related to plant immunity included "activation of innate immune response" (GO:
0002218) (4 genes), "activation of immune response" (GO: 0002253) (4 genes), and "induced systemic resistance, jasmonic acid mediated signaling pathway" (GO: 0009864) (3 genes) (Fig. 6b). In total, 93 enriched KEGG pathways were identified, of which 30 pathways were significantly enriched in this module (Additional file 10: Table_S10). The top 30 significantly enriched pathways for target genes are mentioned in Fig. 7b. "Starch and sucrose metabolism" (ko00500) (14 genes), "Plant hormone signal transduction" (ko04075) (13 genes), "Phenylpropanoid biosynthesis" (ko00940) (11 genes), "Biosynthesis of amino acids" (ko01230) (10 genes), and "DNA replication" (ko03030) (8 genes) were the most significant KEGG pathways.

In the MEbrown2 module, a total of $126 \mathrm{GO}$ terms were assigned, including 89,5 and $32 \mathrm{GO}$ terms in "biological process", "cellular component" and "molecular functions", respectively (Additional file 9: Table_S9). In addition to the terms related to biological metabolism and gene expression regulation, the items related to 

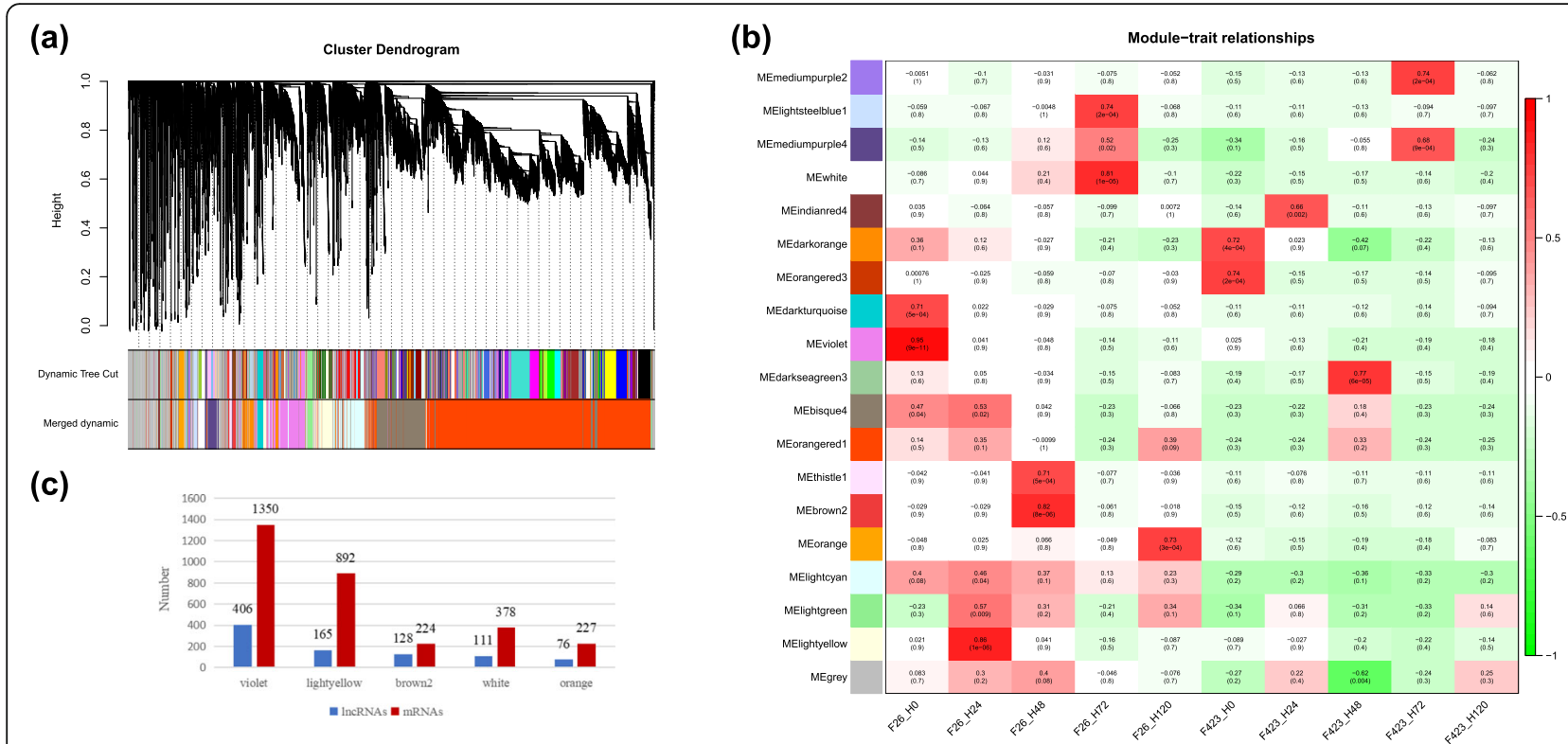

(c)

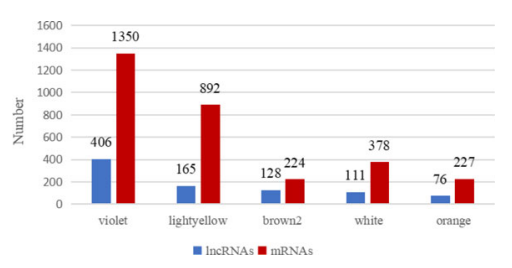

Fig. 5 Weighted gene co-expression network analysis (WGCNA) of IncRNAs in all samples. a Hierarchical cluster tree presenting 19 modules of co-expressed IncRNAs. Each of the 10,645 IncRNAs is represented by a leaf in the tree, with each of the 19 modules presented as a major tree branch. The lower panel provides the modules in distinct colors. $\mathbf{b}$ Heatmaps indicating the correlation of module eigengenes at various infection stages. The Pearson correlation coefficients of each module at various stages are provided and colored according to the score. $\mathbf{c}$ The number of IncRNAs and mRNAs in five significant modules

plant immunity "response to endogenous stimulus" (GO: 0009719) (15 genes), "cellular response to endogenous stimulus" (GO:0071495) (13 genes) and "cellular response to hormone stimulus" (GO:0032870) (12 genes) were also enriched significantly (Fig. 6c). In total, 38 enriched KEGG pathways were identified, of which 30 pathways were significantly enriched in this module (Additional file 10: Table_S10). The top 30 significantly enriched pathways for target genes are mentioned in Fig. 7c. "Cyanoamino acid metabolism" (ko00460) (3 genes), "Plant hormone signal transduction" (ko04075) (6 genes), "Nitrogen metabolism" (ko00910) (2 genes), "Terpenoid backbone biosynthesis" (ko00900) (2 genes) were the most significant KEGG pathways.

In the MEwhite module, a total of $142 \mathrm{GO}$ terms were assigned, including 95, 4 and 43 GO terms in "biological process", "cellular component" and "molecular functions", respectively (Additional file 9: Table_S9). Among the biological process category, the significantly over represented GO terms were "response to stimulus" (GO: 0050896) (67 genes), followed by "response to stress" (GO: 0006950) (51 genes) and "defense response" (GO: 0006952) (43 genes), which were all related to plant immunity. In addition, other terms related to plant immunity were also enriched, such as "immune system process" (GO:0002376) (14 genes), "response to biotic stimulus" (GO:0009607) (14 genes) and "innate immune response" (GO:0045087) (13 genes), etc. (Fig. 6d). In total, 54 enriched KEGG pathways were identified, of which 30 pathways were significantly enriched in this module (Additional file 10: Table_S10). The top 30 significantly enriched pathways for target genes are mentioned in Fig. 7d. "Carbon metabolism" (ko01200) (5 genes), "Cysteine and methionine metabolism" (ko00270) (4 genes), "Amino sugar and nucleotide sugar metabolism" (ko00520) (4 genes) were the most significant KEGG pathways.

In the MEorange module, a total of $128 \mathrm{GO}$ terms were assigned, including 87, 8 and 33 GO terms in "biological process", "cellular component" and "molecular functions", respectively (Additional file 9: Table_S9). Among the biological process category, "response to organic substance" (GO: 0010033) (14 genes), "response to endogenous stimulus" (GO: 0009719) (13 genes), and "response to external stimulus" (GO: 0009605) (10 genes)etc., associated with plant immunity were significantly enriched (Fig. 6e). In total, 32 enriched KEGG pathways were identified, of which 30 pathways were significantly enriched in this module (Additional file 10: Table_S10). The top 30 significantly enriched pathways for target genes are mentioned in Fig. 7e. "Plant hormone signal transduction" (ko04075) (4 genes), "Thiamine metabolism" (ko00730) (3 genes), "Starch and sucrose metabolism" (ko00500) (3 genes) and "Fatty acid degradation" (ko00071) (2 genes) were the most significant KEGG pathways.

\section{Network analysis of hub IncRNAs}

The hub lncRNAs are important for regulating the whole network. Therefore, we screened the 96 hub lncRNAs and 


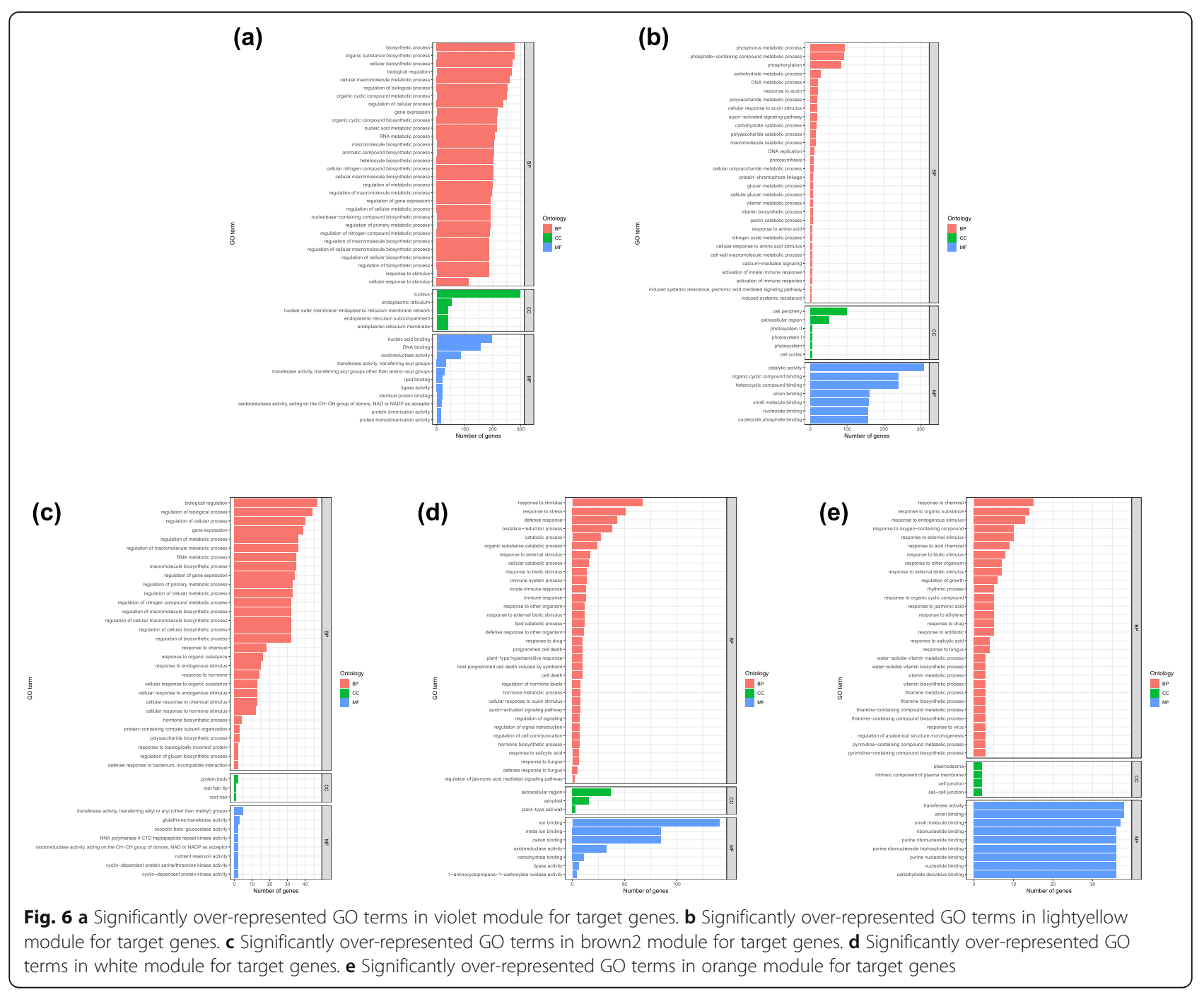

124 known target genes according to their weight value and connectivity in five modules (Additional file 11: Table_S11). In the MEviolet module, the 25 known target genes for 15 hub lncRNAs were found to be involved in multiple functions (Fig. 7a), such as probable galacturonosyl transferase 10 and ultraviolet-B receptor UVR8-like. In addition, target genes encoding receptor-like serine/threonine-protein kinase NCRK (XM_018958556.1) and eukaryotic translation initiation factor 5A-2-like (XM_018994862.1) are known resistance genes (Fig. 8a). In the MElightyellow module, 16 hub lncRNAs were generated and their 22 known target genes were involved in many functions (Fig. 8b). And the target genes encoding G-type lectin S-receptor-like serine/ threonine-protein kinase LECRK1 (XM_018950446.1), probably inactive leucine-rich repeat receptor-like protein kinase At2g25790 (XM_018989953.1) and TMV resistance protein N-like (XM_018961957.1) were known resistance genes (Fig. 8b). In the MEbrown2 module, 24 hub lncRNAs and their 15 known target genes were generated (Fig. 8c), the target gene encoding probable LRR receptor-like serine/threonine-protein kinase At3g47570 (XM_ 018962714.1) was konwn resistance gene (Fig. 8c). In the MEwhite module, 23 hub lncRNAs were generated and their 38 known target genes were involved in many functions (Fig. 8d). The target genes encoding putative disease resistance protein At1g50180 (XM_018965430.1), probable LRR receptor-like serine/ threonine-protein kinase At1g63430 (XM_018973294.1) and L-type lectin-domain containing receptor kinase IV.2like (XM_018954279.1) were konwn resistance genes (Fig. $8 \mathrm{~d})$. In the MEorange module, 18 hub lncRNAs were generated and their 24 known target genes were involved in many functions (Fig. 8e). And the target gene encoding the inactive LRR receptor-like serine / threonine-protein kinase BIR2 (XM_018967526.1) was konwn resistance gene (Fig. 8e). All disease resistance genes in walnut are listed in Additional file 12: Table_S12. These results suggested that lncRNAs may participate in the resistance of 


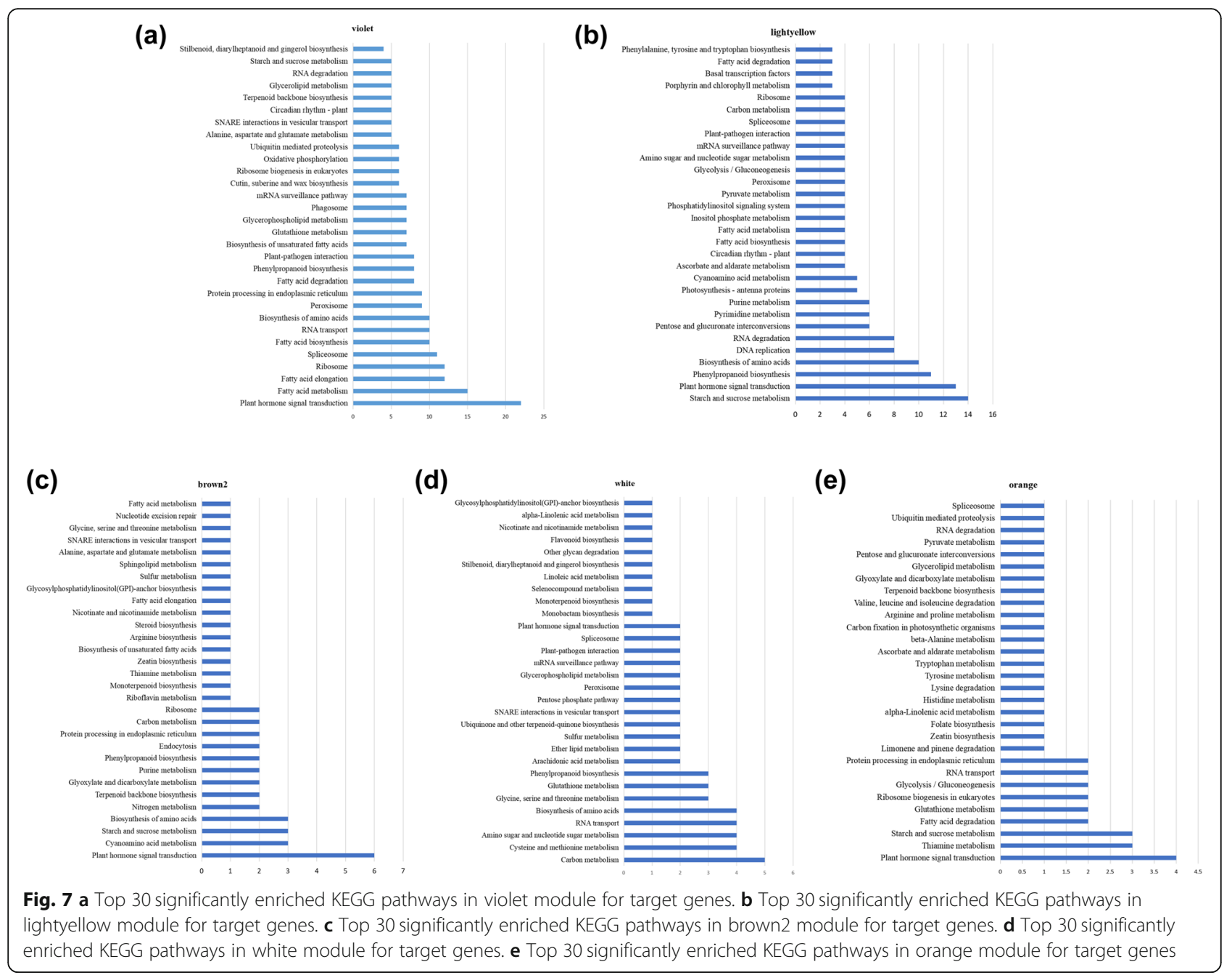

walnut bracts to C. gloeosporioides by acting on their target genes. Based on the enrichment results of KEGG, we predicted the possible pathway of hub lncRNAs (Additional file 13: Table_S13). Most of the hub lncRNAs and its target genes in the five modules are enriched in the pathways of material metabolism and biosynthesis. In the white module, the function of hub lncRNA pathway map showed that cyclicnucleotide-gated channels and MPK4, the target genes of lncRNA MSTRG.94840.7,were upregulated at $72 \mathrm{hpi}$, which were enriched in "plant pathogen interactions" pathway (Fig. 9a). The target genes (SAUR and ABF) of lncRNA103441.8 were involved in "plant hormone signal transduction" pathway,which may be related to plant immunity (Fig. 9b).

\section{Validation of hub IncRNAs and target genes}

We randomly selected 5 hub lncRNAs and 5 target genes for qRT-PCR analysis with the aim to validate the expression profiles between F26 and F423 obtained by
RNA-Seq. The list of hub lncRNAs specific primers used for qRT-PCR analysis is listed in Additional file 14: Table_S14. The hub lncRNAs selected for qRT-PCR confirmation were MSTRG.13585.8, MSTRG.152205.1, MSTRG.11713.16, MSTRG.112028.8, and MSTR G.62751.2, the target genes were related to probable galacturonosyl transferase 10 (LOC109014322), G-type lectin S-receptor-like serine / threonine-protein kinase LECRK1(LOC108979712), NHL repeat-containing (LOC108987880), probable LRR receptor-like serine/ threonine-protein kinase At3g47570 (LOC108989177), and putative disease resistance protein At1g50180 (LOC108991254). The qRT-PCR analysis showed that the expression of MSTRG13585 and LOC109014322 peaked at Ohpi, MSTRG11713, MSTRG152205, LOC108979712 and LOC108987880 at 24hpi, MSTR G112028 and LOC108989177 at 48hpi, MSTRG62751 and LOC108991254 at 72hpi (Fig. 10), which were consistent with the RNA-seq data (Additional file 15: Table_ 

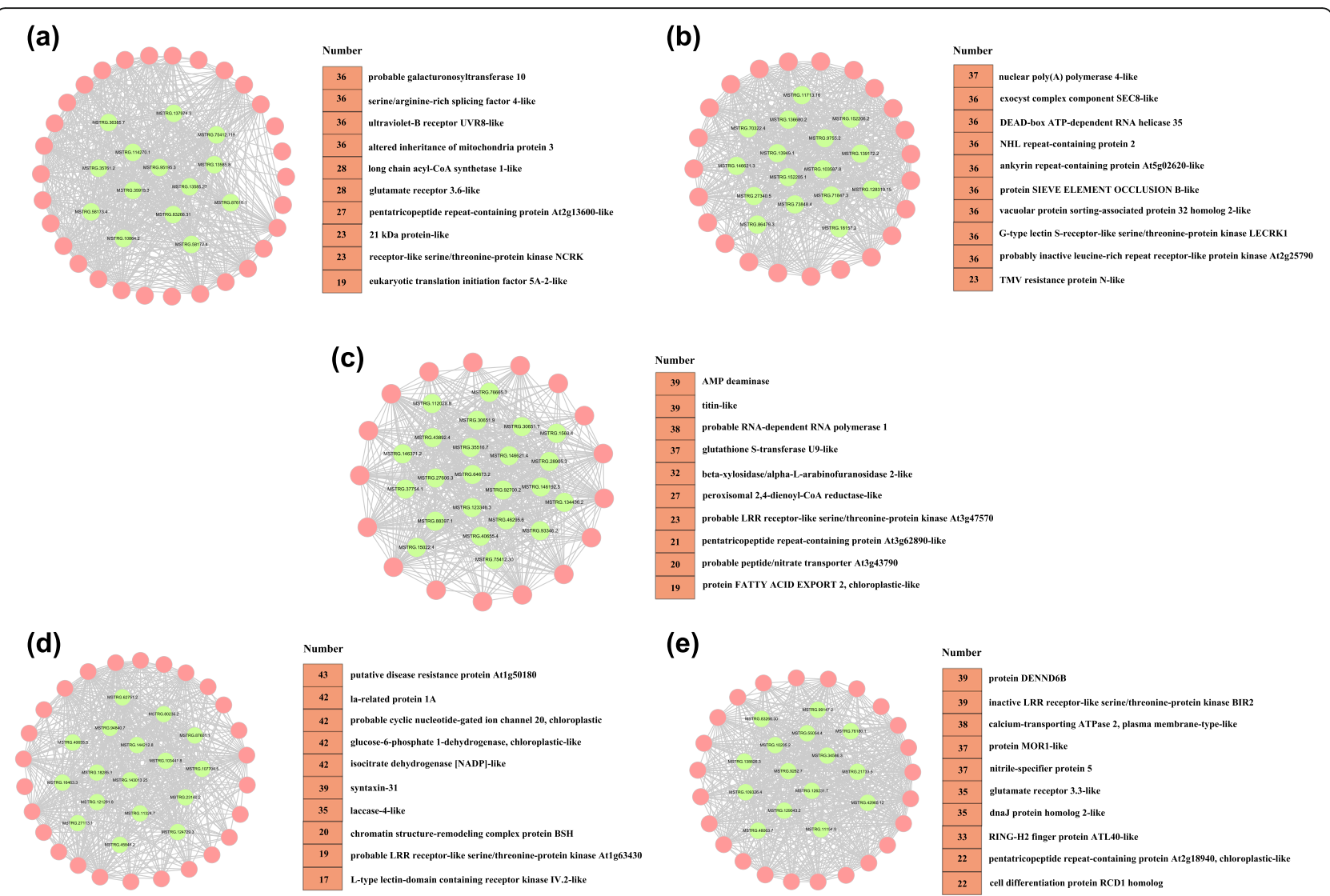

Fig. 8 a Co-expression network associated with violet module. b Co-expression network associated with lightyellow module. c Co-expression network associated with brown2 module. $\mathbf{d}$ Co-expression network associated with white module. e Co-expression network associated with orange module. Red and green represent the target genes (mRNAs) and IncRNAs, respectively. Functional annotation of the target genes of the hub IncRNAs. Numbers represent the number of nodes

S15), with similar trends observed for the hub lncRNAs and their target genes.

\section{Discussion}

In previous studies, lncRNAs were identified and analyzed in various biological processes important for seed development [31], photomorphogenesis [32], fruit development [33, 34], and biotic and abiotic stress responses $[22,35]$. Additionally, there has been substantial research on the role of lncRNAs in plant-pathogen interactions. In A. thaliana, IncRNAs reportedly enhance the resistance to Pseudomonas syringae pv. tomato DC3000 by promoting PR1 expression [36]. In tomato, IncRNA23468 functions as a ceRNA that modulates NBS-LRR gene expression by mimicking the target of miR482b, thereby increasing the resistance to $P$. infestans [37]. Walnut anthracnose has been responsible for the premature fruit drop and yield losses that have adversely affected walnut production in China [13]. In this study, we investigated the role of IncRNAs in the resistance of walnut fruit bracts to anthracnose based on sequence analyses. Walnut anthracnose is caused by $C$. gloeosporioides, which completes its infection process as a hemibiotroph [10,38]. First, conidia germinate to generate appressoria, which produce invasion pegs that initiate the infection into susceptible plants. The primary mycelium produced in plant cells exists as a biotroph, after which the secondary mycelium produced in the infected site switches to necrotrophic growth $[39,40]$. We previously determined that the $C$. gloeosporioides life cycle in walnut tissue involves attachment at $24 \mathrm{hpi}$, biotrophy at $48 \mathrm{hpi}$, and necrotrophy at $72 \mathrm{hpi}$ (data unpublished). In this study, RNA-seq was performed to build the IncRNA and mRNA profiles of the walnut fruit bract tissue at $0 \mathrm{hpi}$, infected tissue at 24,48 , and $72 \mathrm{hpi}$, and distal uninoculated tissue at $120 \mathrm{hpi}$. A total of 58,369 mRNAs and 22,336 lncRNAs were identified, including 3933 known lncRNAs and 18,403 unknown lncRNAs. Consistent with the results of similar studies on other organisms, the identified putative lncRNA had fewer exons, shorter transcripts, and lower expression levels than protein-coding genes [41, 42].

The release of walnut reference genome [1], enabled the study of walnut genetics at a genome-wide scale. 
(a)

\section{plant-pathogen interaction}

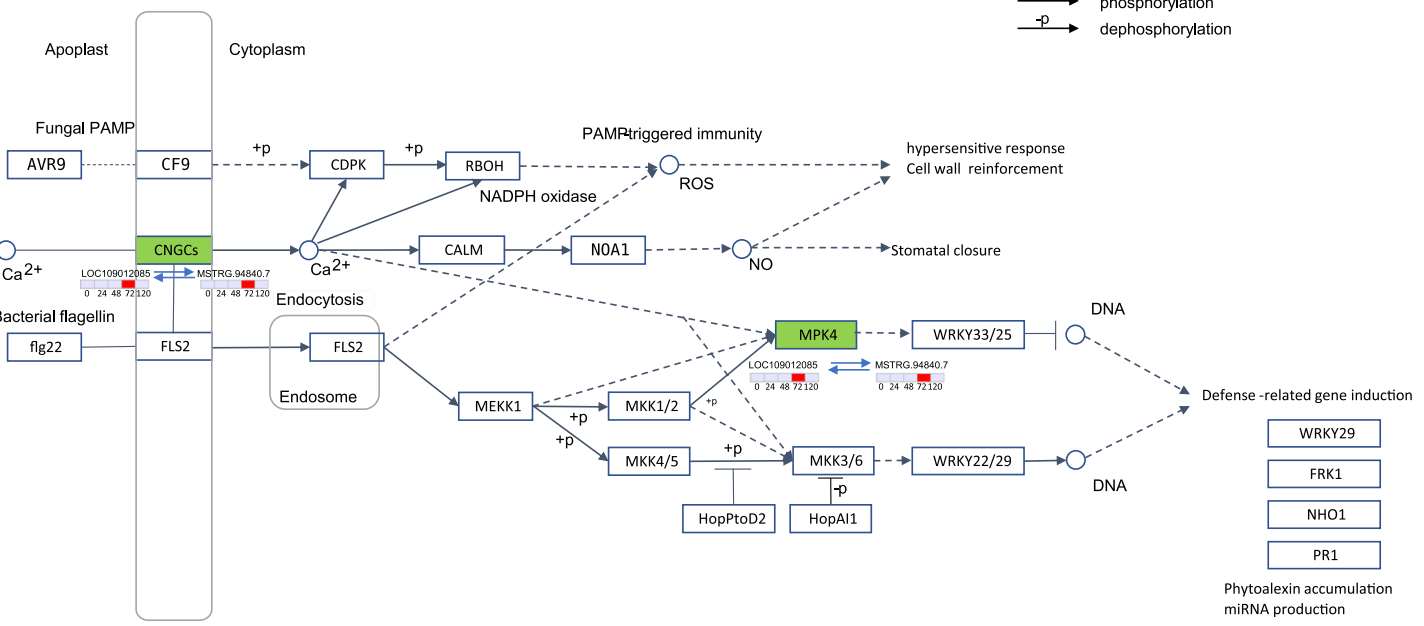

(b)

\section{plant hormone signal transduction}

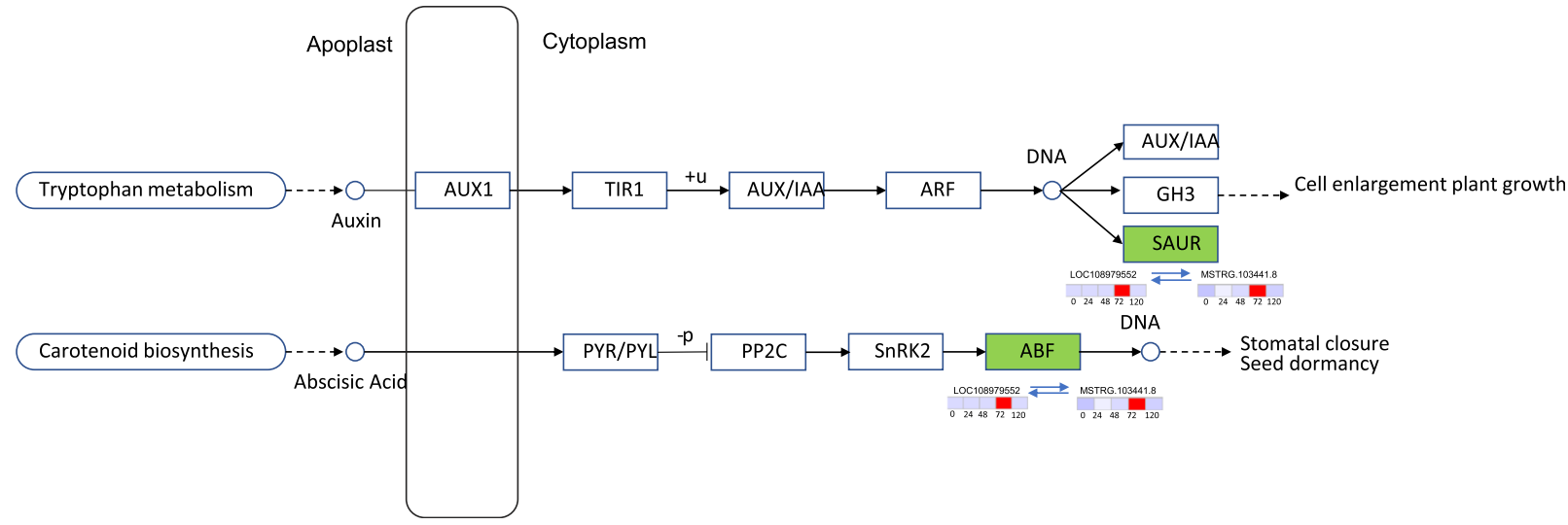

Fig. 9 a MSTRG.94840.7 and the target gene LOC109012085 involved in plant-pathogen interaction pathway. b MSTRG.103441.8 and the target gene LOC108979552 involved in the plant hormone signal transduction pathway enriched by KEGG analysis

Based on the reference genome, the whole-genome resequencing [43], the development of high-density genotyping tools [44], and the genetic dissection of important agronomical traits in walnut [45] have been completed. The development of bioinformatic analysis technology has enabled researchers to reveal that IncRNA functions and characteristics are far more complex than previously thought [16]. A recent comparative transcriptome analysis between wild-type and WRKY1-overexpressing tomato plants revealed 199 lncRNAs (DELs) and indicated that many of the lncRNA target genes that are likely affected by WRKY1 and associated with the resistance of tomato to $P$. infestans are involved in the response to biotic stimulus (GO:0009607) and plant-pathogen 


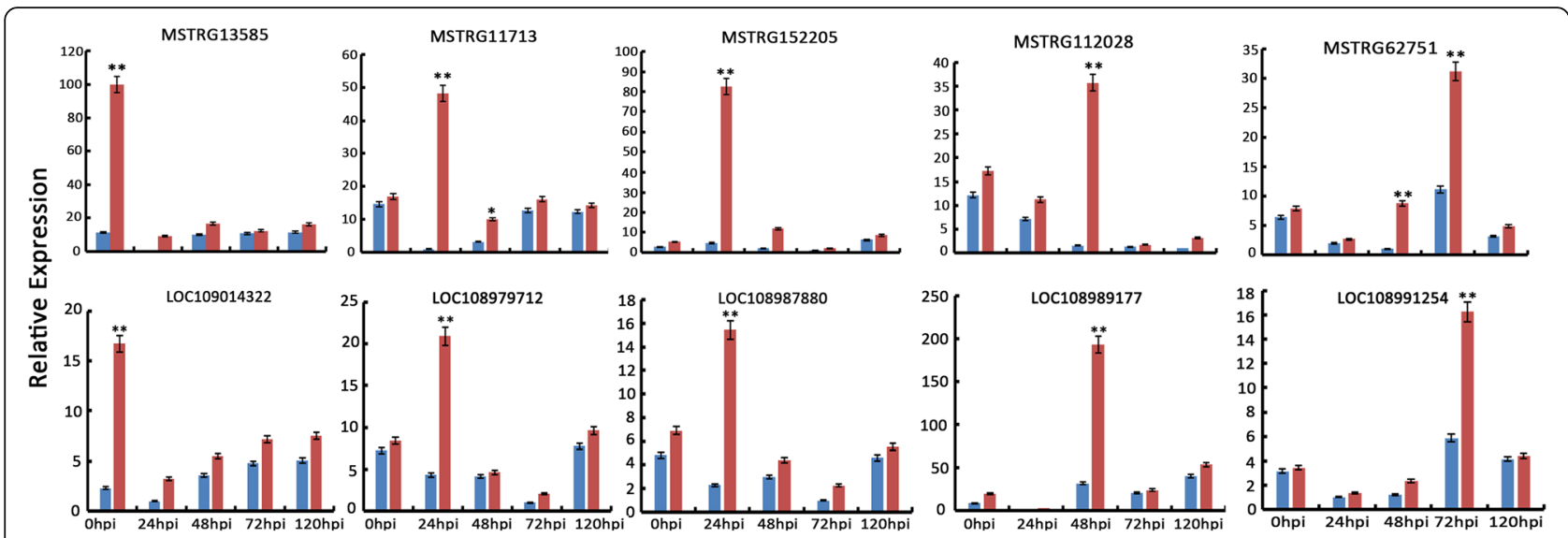

Fig. 10 Validation of selected IncRNAs and mRNAs in a quantitative PCR assay. Blue and red represent the F423 and F26 samples, respectively. Expression data were normalized against the data for the 185 rRNA housekeeping gene and are presented as themean \pm standard error; ${ }^{*} p<0.05,{ }^{* *} p<0.01$

interaction (KO4626) [26]. In another recent study, 4594 putative lncRNAs were identified in comprehensive dynamic lncRNA expression networks under heat stress conditions. Co-expression networks revealing the interactions among the differentially expressed lncRNAs, mRNAs, and microRNAs indicated that several phytohormone pathways are associated with heat tolerance, including salicylic acid and brassinosteroid pathways [21]. In the current study, we obtained 10,645 upregulated lncRNAs and 15,247 upregulated mRNAs among the five comparisons (F26_0hpi vs F423_0hpi, F26_24 hpi vs F423_24 hpi, F26_48 hpi vs F423_48 hpi, F26_72 hpi vs F423_72 hpi, and F26_120 hpi vs F423_120 hpi). The number of up-regulated IncRNAs and mRNAs in the F26 vs F423 was significantly higher at the early stages of C. gloeosporioides infection.

The functions of lncRNAs cannot currently be inferred from their sequence or structure, but lncRNAs can function in trans mode to target gene loci distant from where the lncRNAs are transcribed [46]. In F26, a total of 5 modules related to disease resistance were obtained by WGCNA during the infection of $C$. gloeosporioides. Many target genes of lncRNAs in these modules are enriched in plant immune related items and pathways, such as "activation of innate immune response", "activation of immune response" in MElightyellow module, "defense response to bacterium, incompatible interaction" in MEbrown2 module, "defense response" and "immune system process" in MEwhite module. These results suggest that these genes may play important roles in the process of resistance to C. gloeosporioides of walnut fruit bracts. Phytohormones are known to be important in the regulation of defense responses in plants [47-49]. Plants can exhibit systemic acquired resistance through the salicylic acid (SA) / jasmonic acid (JA)-mediated signaling network [50-53]. In our study, a total of
32 genes were identified in the significantly enriched KEGG pathway "Plant hormone signal transduction". Meanwhile, there are 3 and 5 genes enriched in "jasmonic acid mediated signaling pathway" and "response to jasmonic acid" respectively. We also showed that some genes were enriched in "auxin-activated signaling pathway" and "cellular response to auxin stimulus" at $24 \mathrm{hpi}$. Therefore, auxin may play a role in the resistance of walnut bracts to $C$. gloeosporioides. In addition, our result showed that the phenylpropanoid biosynthesis was one of the most significantly enriched pathways in the process of resistance to C. gloeosporioides of walnut fruit bracts. In this pathway, phenylalanine ammonium lyase (PAL) is the key regulatory enzyme in altering the biosynthesis and accumulation of flavonoids and lignin [54]. Lignin plays a structural role in the secondary cell walls formation [55], and flavonoids mediate plants against UV radiation and act as a visual signal for attracting pollinators [56, 57]. In Caragana korshinskii, C. korshinskii adjusts its phenylpropanoid biosynthesis process to water-deficit environments and activates PAL by drought stress [58].

During long-term evolutionary interactions with plants, several pathogens successfully cause effectortriggered susceptibility response (ETS) by producing a number of effectors. Simultaneously, plants have evolved $\mathrm{R}$ genes that recognize these effectors and function through highly specific interactions between effectors and their corresponding nucleotide-binding site and leucine-rich repeat (NB-LRR) class receptors [59]. In tomato, lncRNA23468 reportedly increases the expression of the NBS-LRR target genes (encoding $\mathrm{R}$ proteins), resulting in enhanced resistance to P.infestans [37]. In the current study, we detected $10 R$ genes among the target genes of 96 hub lncRNAs. During the infection of $C$. gloeosporioides on the walnut fruit bracts, the results of 
RNA-seq showed that the expression of $R$ genes (XM 018950446.1, XM_018989953.1 and XM_018961957.1 in MElightyellow module, XM_018962714.1 in MEbrown2 module, XM_018965430.1, XM_018973294.1 and XM_ 018954279.1 in MEwhite module) were up-regulated at 24hpi, 48hpi and 72hpi respectively, and expression of the highly connected lncRNAs (MSTRG.11713.16, MSTR G.146621.3 and MSTRG.136680.2 in MElightyellow module, MSTRG.123346.3 in MEbrown2 module, MSTR G.94840.7, MSTRG.18285.3 and MSTRG.45846.2 in MEwhite module) had the same trends (Additional file 14 Table_S14). These findings imply that lncRNAs may help mediate the disease resistance of walnut fruit bracts through the $\operatorname{target} R$ genes. The specific interaction between $\operatorname{lncRNAs}$ and $R$ gene needs further verification. The expression levels of five hub lncRNAs (MSTRG13585, MSTRG11713, MSTRG152205, MSTRG112028, and MSTRG62751) and their target genes were further confirmed by qPCR, the results of which were consistent with the RNA-seq data. The data presented here provides researchers with the biological basis for future investigations of the mechanism underlying the disease resistance of walnut fruit bracts.

\section{Conclusions}

In this study we generated the expression profile of lncRNA in anthracnose-resistant F26 and anthracnosesusceptible F423 at five times. Compared with F423, a total of 14,525 DELs were identified, including 10,645 upregulated lncRNAs and 3846 downregulated lncRNAs in F26. Bioinformatic analysis showed that the target genes of upregulated lncRNAs were enriched in immune-related processes, plant hormone signal transduction, phenylpropanoid biosynthesis and other pathways during the infection of C. gloeosporioides. Hub lncRNAs with high connectivity to disease resistant genes were predicted. These results contribute to our understanding of the potential mechanism by which lncRNAs involved in C. gloeosporioides resistance and will facilitate the functional verification of the lncRNA in the future.

\section{Methods}

\section{Plant materials and fungal isolates}

The scions of walnut seedling tree B26 was provided by walnut specialized farmers' cooperative of Dongliugang village, Baishi Town, Wenshang County, Shandong Province, China $\left(35^{\circ} 46^{\prime} 56.2^{\prime \prime} \mathrm{N}, 116^{\circ} 40^{\prime} 30.8^{\prime \prime} \mathrm{E}\right)$. The 4-23 walnut tree was from $\mathrm{F} 1$ progeny of an intraspecific cross between walnut cultivar 'Yuan Lin' (susceptible to anthracnose) $x$ ' Qing Lin' (resistant to anthracnose) which was carried out by ourselves in 2002 . The plant materials were conserved by patch budding onto walnut seedling rootstock at the Forestry Experimental Station of Shandong Agricultural
University, Tai'an, Shandong Province, China ( $36^{\circ} 10^{\prime} 19.2^{\prime \prime}$ $\mathrm{N}, 117^{\circ} 09^{\prime} 1.3^{\prime \prime} \mathrm{E}$ ) in late May 2009. In 2015-2017, we evaluated the anthracnose resistance of each plant for three consecutive years followed by previously described $[8,14]$, and it was found that B26 clone was highly resistant to anthracnose in fruit bract, and the 4-23 clone was highly susceptible to anthracnose in fruit bract. The fruits of B26 clone (i.e., F26) and 4-23 clone (i.e., F423) were used as experimental materials. The voucher specimen of F26 and F423 had been deposited to our lab but not to any publicly available herbarium. We didn't use wild plants in this study and according to national and local legislation, no specific permission was required to collect these plants. C.gloeosporioidesm9 isolates (GenBank ID: GU597322) used in this study were maintained by our group.

\section{Fungal pathogen inoculation of walnut fruits}

Colletotrichum gloeosporioides was cultivated on potato dextrose agar medium for $5-7$ days at $28^{\circ} \mathrm{C}$. To prepare conidial suspensions, the colonies were washed with sterile distilled water containing $0.05 \%(\mathrm{v} / \mathrm{v})$ Tween 80 , passed through a filter (40-100 $\mu \mathrm{m}$ pores), quantified with a hemocytometer, and diluted with sterile distilled water to $105-106$ conidia/ml $[0.001 \%(\mathrm{v} / \mathrm{v})$ Tween 80 final concentration]. Healthy fruits from the east-, south-, and west-facing parts of each tree were collected in mid-June and disinfected with $0.6 \%$ sodium hypochlorite and rinsed with sterile water. The punch inoculation of the detached walnut fruits was completed as previously described [8]. Based on anatomical changes to the infected walnut fruit bract, samples of the inoculation site were collected at $0,24,48$, and $72 \mathrm{hpi}, 0 \mathrm{hpi}$ as a control. Additionally, distal uninoculated tissue was collected at $120 \mathrm{hpi}$. Take two independent samples as biological replicates at each infection time (Additional file 1: Table S1). All samples were flash-frozen in liquid nitrogen and stored at $-80^{\circ} \mathrm{C}$ until analyzed.

\section{Determination of physiological and biochemical data}

The activity of CAT, Chitinase, SOD and contents of $\mathrm{H}_{2} \mathrm{O}_{2}$, salicylic acid and jasmonic acid at five time points of F26 and F423 were determined according to the instructions on the kit. Each sample was repeated three times. The CAT, Chitinase and SOD activity levels were measured and performed according to kit instructions (Solarbio, cat. No. BC0820) and detected by TU-1901 UV Spectrophotometer (Beijing Purkinje General Instrument Co.,Ltd., Beijing). The content of $\mathrm{H}_{2} \mathrm{O}_{2}$, salicylic acid and jasmonic were detected by the Solebao kit (Solarbio, cat. No. BC3595) with microdetermination.

RNA extraction, library construction, and sequencing Total RNA was extracted from F423 and F26 samples with the Thermo Gene JET Plant RNA Purification Mini 
Kit (Thermo Fisher Scientific Inc., USA). The purity and concentration of the extracted RNA were determined with the NanoDrop2000 spectrophotometer (Thermo Fisher Scientific Inc.) $\left(\mathrm{OD}_{260 / 280} \geq 1.8, \mathrm{OD}_{260 / 230} \geq 1.5\right.$, and concentration $>40 \mathrm{ng} / \mu \mathrm{l}$ ). The RNA integrity was assessed by agarose gel electrophoresis. Ribosomal RNA was removed with the Ribo-Zero ${ }^{\mathrm{ma}}$ Magnetic Kit (Epicentre) and the remaining RNA (polyA+ and polyA-) was recovered. The RNA was randomly fragmented to approximately 200-bp sequences in Fragmentation Buffer (Thermo Fisher Scientific Inc.) and then used as the template to synthesize first-strand cDNA with random hexamers. The second cDNA strand was synthesized with dNTPs, RNaseH, and DNA polymerase I. The overhanging ends were filled in with T4 DNA polymerase and Klenow DNA polymerase to generate blunt ends, after which the A base was added to the $3^{\prime}$ end and the fragment was ligated to a linker. The AMPureXP beads were used for selecting fragments. The second cDNA strand containing $U$ was degraded with the USER enzyme, after which a sequencing library was obtained by PCR amplification. A total of 20 sequencing libraries were constructed. The Qubit 2.0 DNA Broad Range Assay (Invitrogen, USA) was used for a preliminary quantification. The sequencing library inserts were detected with the Agilent 2100 Bioanalyzer. Finally, the effective library concentrations $(>2 \mathrm{nM})$ were accurately quantified by qPCR. Paired-end sequencing $(2 \times 150 \mathrm{bp})$ was completed in KeGene Science \& Technology Co. Ltd. (Shandong, China) with an Illumina HiSeq 4000 platform.

\section{Read mapping and transcriptome assembly}

The quality of the raw sequencing data was checked with FASTQC (http://www.bioinformatics.babraham.ac. $\mathrm{uk} /$ projects/fastqc/). Adapters and low-quality tags in the raw data were eliminated. Ribosomal RNA data were also removed. The remaining clean reads for the $20 \mathrm{cDNA}$ libraries were combined and mapped to the J. regia genome sequence (https://www.ncbi.nlm. nih.gov/genome/? term $=$ Juglans $\% 20$ regia) with the HISAT program (version 0.11.5) (parameter setting: -rna-strandness RF) [60]. To construct transcriptomes, the mapped reads were assembled with StringTie (version 1.3.1) [61]. After combining the StringTie results for each sample with StringTieMerge, the read counts were calculated for transcripts with bedtools (version 2.27.1) (bedtools.readthedocs.org /en/latest/\#) [62].

\section{Identification of IncRNAs}

To obtain the potential long non-coding RNAs, based on all the assembled transcripts, we have firstly excluded the known transcripts according to the class code " $="$. Then the remaining transcripts were used to remove the potential protein coding transcripts, miRNA-like, and other transcript types via blasting against the database of Rfam, Refseq, Uniprot, miRbase, and Pfam. Finally, the remaining transcripts were employed for coding potential prediction by using FEELnc tool. First, the FEELnc filter was used to remove short transcripts (default 200 nt) and assess single-exon transcripts [63]. The FEELnc codpot predictors were used to calculate a coding potential score. The assembled sequences were used for reconstructing the transcriptome. Finally, RNAs longer than $200 \mathrm{nt}$ and derived from $\geq 2$ exons, with an ORF coverage $<50 \%$ and a potential coding score $<0.5$ were designated as lncRNAs [64].

\section{Classification of IncRNAs}

The lncRNAs were analyzed regarding their corresponding positions in the reference genome and the positional relationships between lncRNAs and partner RNAs based on 10,000-100,000 fragments. The lncRNAs were then divided into genic lncRNAs (overlapping partner RNAs) and intergenic lncRNAs (lincRNAs). The genic lncRNAs were further divided as overlapping, containing, or nested subtypes. Intergenic lncRNAs were divided as divergent, convergent, and same strand subtypes.

\section{Analysis of differential expression patterns}

Genes differentially expressed between the diseaseresistant and susceptible fruits at five infection stages were analyzed with DESeq2 (version 1.22.1) [65]. After assessing the significance of any differences, the genes with a $p$ value $\leq 0.05$ and a $\mid \log _{2}$ foldchange $\mid \geq 1$ were designated as differentially expressed genes. The principal component analyses (PCA) of F26 and F423 were constructed using the $\operatorname{prcomp}()$ function shipped with the base $\mathrm{R}$ installation. The PCA result was visualized using the ggplot2 package in $\mathrm{R}$.

\section{Quantitative real-time PCR}

Total RNA samples extracted from walnut fruitsat individual infection stages were analyzed by qPCR. Briefly, first-strand cDNA was obtained with the TransScript One-Step gDNA Removal and cDNA Synthesis SuperMixfor qPCR (Transgen, China). The lncRNA expression level was quantified with the TransStart Tip Green qPCR SuperMix (Transgen) and the CFX Connect RealTimeSystem (Bio-Rad). The qPCR program was as follows: $95^{\circ} \mathrm{C}$ for $30 \mathrm{~s} ; 40$ cycles of $95^{\circ} \mathrm{C}$ for $10 \mathrm{~s}$ and $60^{\circ} \mathrm{C}$ for $30 \mathrm{~s}$. For a melting curve analysis, the temperature was increased from $70^{\circ} \mathrm{C}$ to $95^{\circ} \mathrm{C}\left(0.5^{\circ} \mathrm{C} / 5 \mathrm{~s}\right)$. All samples were analyzed in triplicate. The $18 \mathrm{~S}$ rRNA gene was used as a housekeeping gene. The cycle threshold (Ct) $2^{-\Delta \Delta C t}$ method (Software IQ5 2.0) [66] was used for the relative quantification of mRNAs. The primers used for RT-qPCR were designed with Beacon Designer 7 
software and were synthesized by Sangon Biotech (Shanghai, China; Supplementary Table S14).

\section{Prediction of IncRNA functions based on co-expression}

Co-expression modules were generated with the WGCNA package (version 1.67) as previously described [67] (http:// lab.genetics.ucla.edu/horvath/Coexpression Network/). The lncRNAs and mRNAs that were not detected in at least one infection stage were not considered. In this analysis, the soft thresholding power was set to 12, after which the adjacency function was used to construct the adjacency matrix. A topological overlap measure map was constructed based on the adjacency matrix to calculate the similarity matrix of the lncRNA and mRNA expression between different nodes. The lncRNAs and mRNAs were hierarchically clustered based on the algorithm. To generate a number of clusters, modules were defined after eliminating or combining branches. The co-expression module dynamic shear tree parameters were determined as described by Gerttula [68]. The minimum number of genes was set to 30 , the split sensitivity (deep Split) was set to 2, and the other settings were software default parameters. The module was related to the trait, and the correlation matrix between the module and the trait was calculated. The module with the highest correlation coefficient and the smallest $p$ value was designated as the module most relevant to the trait. In this study, a significantly correlated module was identified based on a correlation coefficient $(r) \geq 0.8$ [64] and $p<0.05$. The co-expression networks of lncRNAs and hub lncRNAs in highly correlated modules were generated with the Cytoscape software (version 3.7.1) [69].

\section{Functional enrichment analysis}

The genes targeted by lncRNAs were functionally annotated based on the GO and KEGG pathway (http://www. genome.jp/kegg/) databases. The KOBAS program (version 2.0) was used to determine the significantly enriched KEGG pathways among the target genes [70]. According to the operation requirements of KOBAS 2.0, All data files were written with a parser. The gene-term mapping can be retrieved by parsing the raw data files for each pathway. The gene annotation and gene-ID relations were retrieved from KEGG Genes and BioMart. We mapped the genes in all databases to KEGG GENES and KEGG ORTHOLOGY (KO). The gene-pathway and is stored in our backend SQL relational database. The FASTA protein sequence files were preprocessed for BLAST [71].

\section{Supplementary Information}

The online version contains supplementary material available at https://doi. org/10.1186/s12864-020-07310-6.

Additional file 1: Table S1. Detailed information on ten walnut samples for RNA-seq.
Additional file 2: Table S2. Summary of the sequencing information. Additional file 3: Table S3.The expression level (reads counts) for each IncRNA genes detected in our study.

Additional file 4: Table S4. The expression level (reads counts) for each mRNA genes detected in our study.

Additional file 5: Table S5. TheClassification results of IncRNAs.

Additional file 6: Table S6. All theupregulated and downregulatedlncRNAsamong the five stages.

Additional file 7: Table S7. All theupregulated and downregulatedmRNAsamong the five stages.

Additional file 8: Table S8. The genes information of different modules in the WGCNA.

Additional file 9: Table S9. List of significantly enriched GO terms in five modules.

Additional file 10: Table S10. Summary of KEGG annotations for five modules.

Additional file 11: Table S11. All hub IncRNAs of five significant modules. Genes from the five modules in the F26.

Additional file 12: Table S12. All the disease resistance genes in walnut.

Additional file 13: Table S13. The function of hub IncRNAs pathway. Additional file 14: Table S14. Thesequences of quantitative real time PCR primers.

Additional file 15: Table S15. Expression trend of disease resistance genes and hub IncRNAs.

\section{Abbreviations}

LncRNAs: Long non-coding RNAs; DELs: Different expressed IncRNAs; WGCNA: Weighted Gene Coexpression Network Analysis; hpi: Hours post inoculation; eTM: Endogenous target mimics; ceRNA: Competing endogenous RNA; GO: Gene Ontology; KEGG: Kyoto Encyclopedia of Genes and Genomes; ETS: Effector-triggered susceptibility response; NB-LRR: nucleotide-bindingsite and leucine-rich repeat; ROS: Reactive oxygen species; SA: Salicylic acid; JA: Jasmonic acid

\section{Acknowledgements}

We thank Liwen Bianji, Edanz Editing China (www.liwenbianji.cn/ac) for editing the English text of a draft of this manuscript.

\section{Authors' contributions}

KQY conceived the idea and revised the manuscript. SF and HF conducted meta-analysis, drew figures and drafted the manuscript. XL collected the experimental materials. QW and YD helped in drawing figures and drafting the manuscript. All authors listed have made direct and substantial efforts for improving the manuscript and approved the final version.

\section{Funding}

This study was supported by a Grant from the National Natural Science Foundation of China (No. 31870667 and No. 31470680). The funders had no role in the design of the study, collection, data analysis, data interpretation and manuscript writing.

\section{Availability of data and materials}

The data sets are included within the article and its Additional files. The raw sequencing data were deposited in NCBI Sequence Read Archive under the accession number GSE147083.

(https://www.ncbi.nlm.nih.gov/geo/query/acc.cgi?acc=GSE147083).

Ethics approval and consent to participate

Not applicable.

Consent for publication

Not applicable.

Competing interests

The authors declare that they have no conflicts of interest. 


\section{Author details}

${ }^{1}$ College of Forestry, Shandong Agricultural University, Tai'an 271018, Shandong Province, China. ${ }^{2}$ State Forestry and Grassland Administration Key Laboratory of Silviculture in the Downstream Areas of the Yellow River, Tai'an 271018, Shandong Province, China. ${ }^{3}$ Shandong Taishan Forest Ecosystem Research Station, Tai'an 271018, Shandong Province, China. ${ }^{4}$ Department of Science and Technology, Qingdao Agricultural University, Qingdao 266109, Shandong Province, China.

Received: 16 June 2020 Accepted: 7 December 2020

Published online: 06 January 2021

\section{References}

1. Martínez-García PJ, Crepeau MW, Puiu D, et al. The walnut (Juglans regia) genome sequence reveals diversity in genes coding for the biosynthesis of non-structural polyphenols. Plant J. 2016;87(5):507-32.

2. Bernard A, Lheureux F, Dirlewanger E. Walnut: past and future of genetic improvement. Tree Genet Genomes. 2018;14(1):1.

3. Al-Snafi AE. Chemical constituents, nutritional, pharmacological and therapeutic importance of Juglans regia-a review. IOSR J Pharm. 2018;8(11): $1-21$.

4. Jahanbani R, Ghaffari SM, Salami M, et al. Antioxidant and anticancer activities of walnut (Juglans regia L.) protein Hydrolysates using different proteases [J]. Plant Foods Hum Nutr. 2016;71(4):402-9.

5. Grouh MS, Vahdati K, Lotfi M, et al. Production of haploids in Persian walnut through parthenogenesis induced by gamma-irradiated pollen [J]. J Am Soc Hortic Sci. 2011;136(3):198-204.

6. Arab MM, Marrano A, Abdollahiarpanahi R, et al. Genome-wide patterns of population structure and association mapping of nut-related traits in Persian walnut populations from Iran using the Axiom J. regia 700K SNP array [J]. Sci Rep. 2019;9(1):1-14.

7. Sadat-Hosseini M, Bakhtiarizadeh MR, Boroomand N, et al. Combining independent de novo assemblies to optimize leaf transcriptome of Persian walnut. PLoS One. 2020;15(4):e0232005.

8. An H, Yang K. Resistance gene analogs in walnut (Juglans regia) conferring resistance to Colletotrichum gloeosporioides. Euphytica. 2014;197(2):175-90.

9. Xia $\mathrm{L}$, et al. Laboratory toxicity of eight fungicides against Colletotrichum gloeosporioides causing walnut anthracnose. Chin J Pestic Sci. 2013;15(4):412-20.

10. McDowell JM. Genomic and transcriptomic insights into lifestyle transitions of a hemi-biotrophic fungal pathogen. New Phytol. 2013;197(4):1032-4.

11. Qu WW, Yang KQ, Liu HX, Hou LQ. Main diseases of walnut and integrated management in Shandong. Plant Prot. 2011:44:136-40.

12. O'Connell RJ, Thon MR, Hacquard S, et al. Lifestyle transitions in plant pathogenic Colletotrichum fungi deciphered by genome and transcriptome analyses. Nat Genet. 2012;44(9):1060-5.

13. Lin SY, Okuda S, Ikeda K. LAC2 encoding a secreted laccase is involved in appressorial melanization and conidial pigmentation in Colletotrichum orbiculare. Mol Plant-Microbe Interact. 2012;25(12):1552-61.

14. Zhu Y, Yin Y, Yang K, et al. Construction of a high-density genetic map using specific length amplified fragment markers and identification of a quantitative trait locus for anthracnose resistance in walnut (Juglans regia L. ). BMC Genomics. 2015;16(1):614.

15. An H, Yang K. Sequence characteristics of NBS resistance gene analogues in walnut and their resistance to anthracnose. Agric Sci China. 2014;47(2):344-56.

16. Wang KC, Yang YW, Liu B. A long noncoding RNA maintains active chromatin to coordinate homeotic gene expression. Nature. 2011;472(7341):120-4.

17. Wang HLV, Chekanova JA. Long noncoding RNAs in plants [M]//Long Non Coding RNA Biology. Singapore: Springer; 2017. p. 133-54.

18. Di C, Yuan J, Wu Y. Characterization of stress-responsive IncRNAs in Arabidopsis thaliana by integrating expression, epigenetic and structural features. Plant J. 2014;80:848-61.

19. Ma K, Shi W, Xu M, et al. Genome-wide identification and characterization of long non-coding RNA in wheat roots in response to $\mathrm{Ca}^{2+}$ channel blocker. Front Plant Sci. 2018;9:244.

20. Huanca-Mamani W, Arias-Carrasco R, Cárdenas-Ninasivincha S, et al. Long non-coding RNAs responsive to salt and boron stress in the hyper-arid Lluteno maize from Atacama Desert. Genes. 2018;9(3):170.

21. Wang A, Hu J, Gao C, et al. Genome-wide analysis of long non-coding RNAs unveils the regulatory roles in the heat tolerance of Chinese cabbage (Brassica rapa ssp. chinensis). Sci Rep. 2019;9(1):5002.
22. Qin T, Zhao H, Cui P, et al. A nucleus-localized long non-coding RNA enhances drought and salt stress tolerance. Plant Physiol. 2017;175:1321-36.

23. Heo JB, Sung S. Vernalization-mediated epigenetic silencing by a long intronic noncoding RNA. Science. 2011;331(6013):76-9.

24. Swiezewski S, Liu F, Magusin A, et al. Cold-induced silencing by long antisense transcripts of an Arabidopsis Polycomb target. Nature. 2009; 462(7274):799.

25. Wang Z, Zhai X, Cao Y, et al. Long non-coding RNAs responsive to witches' broom disease in Paulownia tomentosa. Forests. 2017;8(9):348.

26. Cui J, Luan $\mathrm{Y}$, Jiang $\mathrm{N}$, et al. Comparative transcriptome analysis between resistant and susceptible tomato allows the identification of IncRNA16397 conferring resistance to Phytophthora infestans by co-expressing glutaredoxin. Plant J. 2017;89:577-89.

27. Cui J, Jiang N, Meng J, et al. LncRNA33732-respiratory burst oxidase module associated with WRKY1 in tomato-Phytophthora infestans interactions. Plant J. 2019;97(5):933-46.

28. Zhang L, Wang M, Li N, et al. Long noncoding RNAs involve in resistance to Verticillium dahliae, a fungal disease in cotton. Plant Biotechnol J. 2018;16(6): $1172-85$.

29. Xin M, Wang Y, Yao Y, et al. Identification and characterization of wheat long non-protein coding RNAs responsive to powdery mildew infection and heat stress by using microarray analysis and SBS sequencing. BMC Plant Biol. 2011;11(1):61.

30. Zhang $\mathrm{H}$, Chen $\mathrm{X}$, Wang $\mathrm{C}$, et al. Long non-coding genes implicated in response to stripe rust pathogen stress in wheat (Triticumae stivum L.). Mol Biol Rep. 2013;40(11):6245-53.

31. Kiegle EA, Garden A, Lacchini E, et al. A genomic view of alternative splicing of long non-coding RNAs during rice seed development reveals extensive splicing and IncRNA gene families. Front Plant Sci. 2018;9:115.

32. Wang H, Chung PJ, Liu J, et al. Genome-wide identification of long noncoding natural antisense transcripts and their responses to light in Arabidopsis. Genome Res. 2014;24(3):444-53.

33. Zhu B, Yang $Y$, Li R, et al. RNA sequencing and functional analysis implicate the regulatory role of long non-coding RNAs in tomato fruit ripening. J Exp Bot. 2015;66:4483-95.

34. Li R, Fu D, Zhu B, et al. CRISPR/Cas9-mediatedmutagenesis of IncRNA1459 alters tomato fruit ripening. Plant J. 2018;94:513-24.

35. Wang J, Yang $Y$, Jin $L$, et al. Re-analysis of long non-coding RNAs and prediction of circRNAs reveal their novel roles in susceptible tomato following TYLCV infection. BMC Plant Biol. 2018;18:104.

36. Seo JS, Sun HX, Park BS, et al. ELF18-INDUCED LONG-NONCODING RNA associates with mediator to enhance expression of innate immune response genes in Arabidopsis. Plant Cell. 2017;29(5):1024-38.

37. Jiang N, Cui J, Shi Y, et al. Tomato IncRNA23468 functions as a competing endogenous RNA to modulate NBS-LRR genes by decoying miR482b in the tomato-Phytophthora infestans interaction. Horticulture Res. 2019;6:28.

38. Da Lio D, Cobo-Díaz JF, Masson C, et al. Combined Metabarcoding and multi-locus approach for genetic characterization of Colletotrichum species associated with common walnut (Juglans regia) anthracnose in France. Sci Rep. 2018;8(1):10765.

39. Alkan N, Friedlander G, Ment D, et al. Simultaneous transcriptome analysis of Colletotrichum gloeosporioides and tomato fruit pathosystem reveals novel fungal pathogenicity and fruit defense strategies. New Phytol. 2015; 205:801-15.

40. Gan P, Ikeda K, Irieda H, et al. Comparative genomic and transcriptomic analyses reveal the hemibiotrophic stage shift of Colletotrichum fungi. New Phytol. 2013;197(4):1236-49.

41. Cabili MN, Trapnell C, Goff L, et al. Integrative annotation of human large intergenic noncoding RNAs reveals global properties and specific subclasses. Genes Dev. 2011;25(18):1915-27.

42. Pauli A, Valen E, Lin MF, et al. Systematic identification of long noncoding RNAs expressed during zebrafish embryogenesis. Genome Res. 2012;22(3): 577-91.

43. Zhang $B, X u L$, Li N, et al. Phylogenomics reveals an ancient hybrid origin of the Persian walnut. Mol Biol Evol. 2019;4:msz112.

44. Marrano A, Martínez-García PJ, Bianco L, et al. A new genomic tool for walnut (Juglans regia L.): development and validation of the high-density AxiomTM J. regia 700 K SNP genotyping array. Plant Biotechnol J. 2018; 17(6):1027-36. 
45. Marrano A, Sideli GM, Leslie CA, et al. Deciphering of the genetic control of phenology, yield and pellicle color in Persian walnut (Juglans regia L.). front. Plant Sci. 2019;10:1140.

46. Nie M, Deng ZL, Liu J, Wang DZ. Noncoding RNAs, emerging regulators of skeletal muscle development and diseases. Biomed Res Int. 2015;2015:1-17.

47. Mao YB, Liu YQ, Chen DY, et al. Jasmonate response decay and defense metabolite accumulation contributes to age-regulated dynamics of plant insect resistance. Nat Commun. 2017;8:13925.

48. Betsuyaku S, Katou S, Takebayashi Y, et al. Salicylic acid and jasmonic acid pathways are activated in spatially different domains around the infection site during effector-triggered immunity in Arabidopsis thaliana. Plant and Cell Physiol. 2017;59(1):8-16.

49. Berens ML, Berry HM, Mine A, et al. Evolution of hormone signaling networks in plant defense. Annu Rev Phytopathol. 2017;55:401-25.

50. Panstruga R, Parker JE, Schulze-Lefert P. SnapShot: Plant immune response pathways. Cell. 2009;136(5):978 e1-3.

51. Muthamilarasan M, Prasad M. Plant innate immunity: An updated insight into defense mechanism. J Biosci. 2013;38(2):433-49.

52. Spoel SH, Dong X. How do plants achieve immunity? Defence without specialized immune cells. Nat Rev Immunol. 2012;12(2):89-100.

53. Dodds PN, Rathjen JP. Plant immunity: towards an integrated view of plantpathogen interactions. Nat Rev Genet. 2010;11(8):539-48.

54. Dai LP, Xiong ZT, Huang Y, Li MJ. Cadmium-induced changes in pigments, total phenolics, and phenylalanine ammonia-lyase activity in fronds of Azolla imbricata. Environ Toxicol. 2006;21(5):505-12.

55. Vanholme R, Demedts B, Morreel K, et al. Lignin biosynthesis and structure. Plant Physiol. 2010;153:895-905.

56. Mol J, Grotewold E, Koes R. How genes paint flowers and seeds. Trends Plant Sci. 1998;3:212-7.

57. Bradshaw HD, Schemske DW. Allele substitution at a flower colour locus produces a pollinator shift in monkeyflowers. Nature. 2003;426:176-8.

58. Liu F, Xie L, Yao Z, et al. Caragana korshinskii phenylalanine ammonialyase is up-regulated in the phenylpropanoid biosynthesis pathway in response to drought stress. Biotechnol Biotechnol Equip. 2019;33(1):842-54.

59. Zhang D, Bao Y, Sun Y, et al. Comparative transcriptome analysis reveals the response mechanism of $\mathrm{Cf}$-16-mediated resistance to Cladosporium fulvum infection in tomato. BMC Plant Biol. 2020;20:30.

60. Kim D, Langmead B, Salzberg SL. HISAT: a fast spliced a gner with low memory requirements. Nat Methods. 2015;12(4):357.

61. Pertea M, Pertea GM, Antonescu CM, et al. StringTie enables improved reconstruction of a transcriptome from RNA-seq reads. Nat Biotechnol. 2015; 33(3):290.

62. Quinlan AR, Hall IM. BEDTools: a flexible suite of utilities for comparing genomic features. Bioinformatics. 2010;26(6):841-2.

63. Harrow J, Frankish A, Gonzalez JM, et al. GENCODE: the reference human genome annotation for the ENCODE project. Genome Res. 2012;22(9):1760-74.

64. Zhao X, Gan L, Yan C, et al. Genome-wide identification and characterization of long non-coding RNAs in Peanut. Genes. 2019:10(7):536.

65. Love Ml, Huber W, Anders S. Moderated estimation of fold change and dispersion for RNA-seq data with DESeq2. Genome Biol. 2014;15(12):550.

66. Livak KJ, Schmittgen TD. Analysis of relative gene expression data using real-time quantitative PCR and the 2(-Delta Delta $C(T))$ method. Methods. 2001;25(4):402-8

67. Langfelder $\mathrm{P}$, Horvath S. WGCNA: an R package for weighted correlation network analysis. BMC Bioinform. 2008:9(1):559.

68. Gerttula S, Zinkgraf M, Muday GK, et al. Transcriptional and hormonal regulation of gravitropism of woody stems in Populus. Plant Cell. 2015; 27(10):2800-13.

69. Jiang $\mathrm{H}, \mathrm{Ma}$ R, Zou S, et al. Reconstruction and analysis of the IncRNAmiRNA-mRNA network based on competitive endogenous RNA reveal functional IncRNAs in rheumatoid arthritis. Mol BioSyst. 2017;13(6):1182-92.

70. Kong L, Zhang Y, Ye ZQ, et al. CPC: assess the protein-coding potential of transcripts using sequence features and support vector machine. Nucleic Acids Res. 2007;35(2):W345-9.

71. Xie C, Mao X, Huang J, et al. KOBAS 2.0: a web server for annotation and identification of enriched pathways and diseases. Nucleic Acids Res. 2011;39: W316-22.

\section{Publisher's Note}

Springer Nature remains neutral with regard to jurisdictional claims in published maps and institutional affiliations.

\section{Ready to submit your research? Choose BMC and benefit from:}

- fast, convenient online submission

- thorough peer review by experienced researchers in your field

- rapid publication on acceptance

- support for research data, including large and complex data types

- gold Open Access which fosters wider collaboration and increased citations

- maximum visibility for your research: over $100 \mathrm{M}$ website views per year

At BMC, research is always in progress.

Learn more biomedcentral.com/submissions 